\title{
Novel Approaches of Evaluating Texture based Similarity Features for Efficient Medical Image Retrieval System
}

\author{
N.Gnaneswara Rao \\ Research Scholar, JNT University: \\ Kakinada, \\ Associate Professor, Dept of CSE, \\ Gudlavalleru Engg. College, \\ Gudlavalleru, A.P., India.
}

\author{
Dr.V.VijayaKumar \\ Prof. \& Dean Dept of Computer \\ Sciences, \\ Head Srinivasa Ramanujan \\ Research Forum, \\ Godavari Institute of Engg \& Tech., \\ Rajahmundry, A.P., India
}

\author{
Dr.P.S.V.Srinivasa Rao \\ Professor, Dept of CSE \\ Rao \& Naidu Engg. College, \\ Ongole, A.P. India
}

\begin{abstract}
The digital medical images in real world are intensity images that carry less information, more noise, less resolution and poor illumination levels. In such cases the existing retrieval system fails in retrieving the relevant images. This is because the chosen similarity features in those systems are not effective for the above types of image retrieval. To address these issues the present paper proposed new similarity texture feature derived from the novel idea of Basic Texture Unit (BTU), Reduced Texture Unit (RTU) and Fuzzy based Texture Unit (FTU). The Texture Unit (TU) extracts textural information of an image with a more complete respect of texture characteristics in all the eight directions instead of only one displacement vector. In most of the real images two neighboring pixel may not have the same value due to the different processes of capture, illumination levels, poor resolutions or digitations. This criterion is met in the proposed BTS, RTS and FTS derived from BTU, RTU and FTU respectively. The BTU, RTU and FTU gives only ternary ,binary and five values respectively to a texture element and TU ranges 0 to 6561,0 to 255 and 0 to 2020 respectively. The similarity features are extracted on BTU, RTU and FTU schemes and a good comparison is made. The experimental results on MRI and Orthopedics images indicate reliability, feasibility and efficacy of the proposed methods.
\end{abstract}

\section{Keywords}

Medical imaging, texture, texture spectrum, similarity feature, image retrieval.

\section{INTRODUCTION}

Texture is an important spatial feature useful for identifying objects or regions of interest in an image.In the past many texture based methods are developed for extracting features of medical images. They yield high level interpretation by providing quantitative information on medical images. Texture is one of the crucial primitives in human vision and texture features have been used to identify content of images. Texture refers to the visual patterns that have properties of homogeneity that do not result from the presence of only a single color or intensity. Texture contains important information about the structural arrangement of surfaces and their relationship to the surrounding environment.

One crucial distinction between color and texture features is that color is a point, or pixel, property, whereas texture is a local-neighborhood property. As a result, it does not make any sense to discuss the texture content at pixel level without considering the neighborhood. Texture has long been an important topic in image processing [1,2,3,4,5,6,7,8,9,10,11 and 12].

Medical images have become the recent key investigation tools for medical diagnosis and treatment planning. Due to the advent of digital imaging the need of data storage and retrieval of medical images increased rapidly. Some difficulties in retrieving the medical images are: medical images have only intensity images that carry less information, more noise, with poor resolution and illumination levels. Therefore the records of medical images are large and complex to analyze. To address this intensified problem of medical image retrieval, the present paper used the proposed novel approach of retrieval system on medical images. The increasing reliance of modern medicine on diagnostic techniques such as radiology, Computerized Tomography (CT) has resulted in an explosion in the number and importance of medical images [13,14,18]. Medical Image acquisition devices such as Computed Tomography (CT) scanners, Magnetic Resonance Imagers (MRI), Ultrasound probes (Us) provide images with various properties in terms of resolution, contrast and signal to noise ratio. They also produce images with different information on the human body anatomy and physiology $[15,16]$. Development of Medical image indexing and retrieval tools is difficult because of the following three factors such as

1. In most cases, medical images are only intensity images carrying less information than colour images.

2. Medical images are usually of low resolution and high noise images. They are difficult to analyze for extracting features automatically. Medical images 
acquired with different devices, even using the same modality, may have significantly varying properties. Some authors proposed image correction and normalization algorithms to improve image comparison.

3. Ideally, medical images should be indeed on medical criteria that are extremely variable depending on the kind of image acquisition considered (imaged body area, clinical context, etc).

As a consequence, medical CBIRs require a high level of content understanding and interpretation of images, which is possible by exploring texture features. Finally, a high level of query completion and accuracy is required by such systems to make them reliable from a clinical point of view [17].

To address these issues the present paper considered three efficient texture based similarity features on Integrated Histogram Bin Matching (IHBM) Similarity Measures [19] for efficient image retrieval. The similarity measures IHBM is applied on the extracted texture similarity features derived from BTS, RTS, and FTS. The results are compared for the query image with database images [20]. The principle of the similarity measure is the computation of the distance between the extracted texture similarity features of the query image and those of the images in the database. Once all the distances are computed, the IHBM similarity measures gives the top 5 or top 10 relevant images as required by the user, which are very nearer to the query image[20].

The present paper is organized as follows. The section two describes the three novel texture similarity features. Section three describes the experimental results and section four describes the conclusions.

\section{PROPOSED TEXTURE BASED SIMILARITY FEATURES FOR EFFICIENT MEDICAL IMAGE RETRIEVAL SYSTEM}

For building effective image retrieval system, especially with images of noise, poor resolution and illumination levels, the most important and crucial factor is selection of the appropriate and efficient similarity feature. For this the present paper has considered three novel approaches called BTU of evaluating texture based similarity features for efficient medical image retrieval system.

\subsection{Basic Texture Spectrum (BTS)}

The texture image can be decomposed into a set of essential small units, called Texture Units (TU). As the texture unit represents the local texture aspect, the statistics of Texture Units in an image should reveal its texture information. The occurrence distribution of Texture Units is called as Texture Spectrum (TS), with the 'abscissa' indicating the type of Texture Unit and the 'ordinate' representing its occurrence frequency.

This section gives a brief review of the Texture Unit (TU) and proposes different methods of calculating texture unit, from which texture spectrum will be constructed. The TU is introduced and described in detail by D.C. He and Li Wang $[3,4,12]$. The basic concept is that a texture image can be considered as a set of essential small units termed as texture units, which characterize the local texture information for a given pixel and its neighborhood.

In a square-raster digital image, each pixel is surrounded by eight neighboring pixels. The local texture information for a pixel can be extracted from a neighborhood of $3 \times 3$ pixels, which represents the smallest complete unit (in the sense of having eight directions surrounding the pixel). A neighborhood of $3 \times 3$ pixels is denoted by a set containing nine elements: $V=\left\{V_{0}, V_{l}\right.$ $\left.\ldots V_{8}\right\}$, here $\mathrm{V}_{0}$ represents the intensity value of the central pixel and $\mathrm{V}_{i}\{\mathrm{i}=1,2 \ldots 8\}$, is the intensity value of the neighboring pixel i. Based on this the corresponding TU is defined by a set containing eight elements. $\mathrm{TU}=\left\{\mathrm{E}_{1}, \mathrm{E}_{2} \ldots \mathrm{E}_{8}\right\}$, where $\mathrm{E}_{i}(\mathrm{i}=1$, $2 \ldots .8)$ is determined by the formula $[4,12]$ given in equation 1 and the element $\mathrm{E}_{i}$ occupies the same position as the pixel $\mathrm{i}$.

$$
\mathrm{E}_{\mathrm{i}}=\left\{\begin{array}{lll}
0 & \text { if } & \mathrm{V}_{\mathrm{i}}<\mathrm{V}_{0} \\
1 & \text { if } & \mathrm{V}_{\mathrm{i}}=\mathrm{V}_{0} \\
2 & \text { if } & \mathrm{V}_{\mathrm{i}}>\mathrm{V}_{0}
\end{array} \text { for }: \mathrm{i}=1,2, \ldots 8\right\} .
$$

The basic texture units are labeled by using the following equation 2, as also represented in Fig.1. As each element of TU has one of the three possible values, the combination of all the eight elements results in $3^{8}=6561$ possible texture units in total. The TU that ranges from o to 6561 is named as Basic Texture Unit (BTU) in the present paper. The texture elements can be ordered clockwise around the centre pixel. The first element may take eight possible positions from the top left corner to the left middle. There is no unique way to label and order the 6561 texture units. These texture units are labeled and ordered in different ways.

$\mathrm{N}_{\mathrm{TU}}=\sum_{\mathrm{i}=1}^{8} \mathrm{E}_{\mathrm{i}} \times 3^{\mathrm{i}-1}, \mathrm{~N}_{\mathrm{TU}} \in\left\{0,1, \ldots \ldots .\left(3^{8}-1\right)\right\}$

\begin{tabular}{|l|l|l|}
\hline $\mathrm{V}_{1}$ & $\mathrm{~V}_{2}$ & $\mathrm{~V}_{3}$ \\
\hline $\mathrm{V}_{8}$ & $\mathrm{~V}_{0}$ & $\mathrm{~V}_{4}$ \\
\hline $\mathrm{V}_{7}$ & $\mathrm{~V}_{6}$ & $\mathrm{~V}_{5}$ \\
\hline
\end{tabular}$\quad$\begin{tabular}{|l|l|l|}
\hline $\mathrm{E}_{1} \times 3^{0}$ & $\mathrm{E}_{2} \times 3^{1}$ & $\mathrm{E}_{3} \times 3^{2}$ \\
\hline $\mathrm{E}_{7} \times 3^{6}$ & $\mathrm{E}_{6} \times 3^{5}$ & $\mathrm{E}_{5} \times 3^{4}$ \\
\hline
\end{tabular}

Fig 1: Representation of Texture Elements

The occurrence distribution of BTU is called as Basic Texture Spectrum (BTS), with the 'abscissa' indicating the type of BTU and the 'ordinate' representing its occurrence frequency.

\subsection{Reduced Texture Spectrum (RTS)}

In RTU [21], the texture unit is defined by the following equation 3 . 


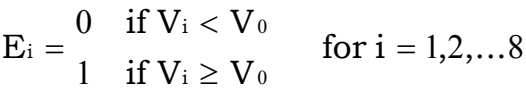

and the element $\mathrm{E}_{i}$ occupies the same position as the pixel $i$.

Since each element of TU in the present method has one of the two possible values, the combination of all the eight elements results in $2^{8}=256$ possible texture units in total. There is no unique way to label and order the 256 texture units. The 256 texture units are labeled by using the following equation 4 :

$\mathrm{N}_{\mathrm{TU}}=\sum_{\mathrm{i}=1}^{8} \mathrm{E}_{\mathrm{i}} \times 2^{\mathrm{i}-1}, \mathrm{~N}_{\mathrm{TU}} \in\left\{0,1, \ldots \ldots\left(2^{8}-1\right)\right\}$.

Here $\mathrm{N}_{\mathrm{TU}}$ represents the texture unit number and $\mathrm{E}_{\mathrm{i}}$ is the $\mathrm{i}^{\text {th }}$ element of texture unit set $\mathrm{TU}=\left\{\mathrm{E}_{1}, \mathrm{E}_{2} \ldots \mathrm{E}_{8}\right\}$. An example of proposed TU is given below in Fig 2 .

\begin{tabular}{|l|l|l|}
\hline 63 & 28 & 45 \\
\hline 88 & 40 & 35 \\
\hline 67 & 40 & 21 \\
\hline
\end{tabular}

(a)

\begin{tabular}{|l|l|l|}
\hline $2^{0}$ & $2^{1}$ & $2^{2}$ \\
\hline $2^{7}$ & & $2^{3}$ \\
\hline $2^{6}$ & $2^{5}$ & $2^{4}$ \\
\hline
\end{tabular}

(c)

\begin{tabular}{|l|l|l|}
\hline 1 & 0 & 1 \\
\hline 1 & & 0 \\
\hline 1 & 1 & 0 \\
\hline
\end{tabular}

(b)

\begin{tabular}{|l|l|l|}
\hline 1 & 0 & 2 \\
\hline 128 & & 0 \\
\hline 64 & 32 & 0 \\
\hline
\end{tabular}

(d)
Fig 2: Transformation model of a neighborhood to a Texture Unit by the proposed RTS. : 2(a) Sample Grey level Neighborhood, (b) Conversion of Fig. 2(a) into Binary Neighborhood, (c) Representation of Binary Weights of TU, (d) Represented Values with Binary Weights.

Fig. 2 shows an example on how to compute TU. The original $3 \times 3$ neighborhood is given in Fig. 2(a). The central pixel value is used as a threshold in order to assign a binary value to its neighbors. Fig. 2(b) shows the result after the thresholding the $3 \times 3$ neighborhood, with central pixel as specified in equation 3 . The obtained values are multiplied by their corresponding weights as shown by Fig. 2(c). The result is given in Fig. 2(d). The sum of the resulting values gives the TU which is 227 in this case. Therefore the central pixel 40 is replaced by the obtained TU value 227. The occurrence distribution of RTU is called as Reduced Texture Spectrum (RTS) [21], with the 'abscissa' indicating the type of RTU and the 'ordinate' representing its occurrence frequency.

\subsection{Fuzzy based Texture Spectrum (FTS): A New Similarity feature for an efficient image retrieval system}

The previous approaches of texture units were unable to discriminate the differences from less, far less or greater and far greater than from the grey level value of central pixel. To incorporate this type of texture feature on a $3 \times 3$ mask, the present paper extends the concept of RTU, features to FTU.

Given a neighborhood of $3 \times 3$ pixels denoted by a set of nine elements: $\quad \mathrm{V}=\left\{\mathrm{V}_{0}, \mathrm{~V}_{1}, \mathrm{~V}_{2}, \ldots \mathrm{V}_{8}\right\}$ where $\mathrm{V}_{0}$ represents the intensity value of central pixel and $\mathrm{V}_{0}, \mathrm{~V}_{1}, \mathrm{~V}_{2}, \ldots \mathrm{V}_{8}$ are the intensity values of eight neighboring pixels.

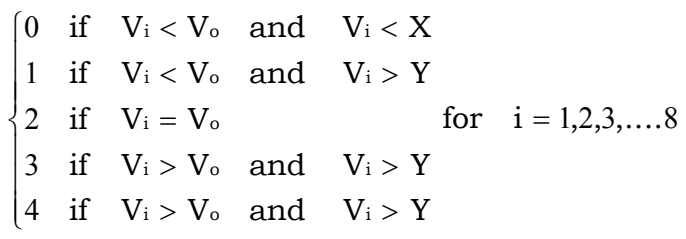

Where $\mathrm{x}, \mathrm{y}$ are user-specified values.

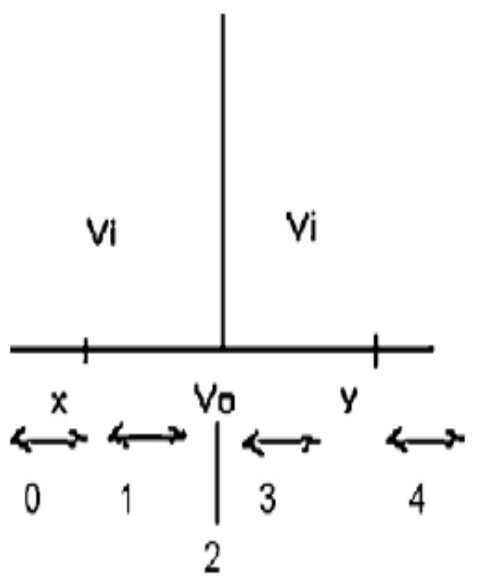

Fig 3: Texture number (Base5) representation.

The corresponding texture unit can be represented as a set containing eight elements, $\mathrm{TU}=\left\{\mathrm{E}_{1}, \mathrm{E}_{2}, \ldots \mathrm{E}_{8}\right\}$. The fuzzy texture membership function is represented as shown in Fig. 3. In Base5, the following Eq. 5 is used determine the elements, $\mathrm{E}_{\mathrm{i}}$ of texture unit. The Fuzzy Texture Unit (FTU) is computed in Base 5 as given in Eq. 6. The FTU ranges from 0 to 2020 as per equation 6

$$
\text { FT nu } 5=\sum_{\mathrm{i}=1}^{8} \mathrm{E}_{\mathrm{i}} \times 5^{(\mathrm{i}-1 / 2)}
$$

Therefore the range of FTU ranges in between BTU and RTU . For example the transformation model of a $3 \times 3$ neighborhood in to FTU is shown in Fig 4. 


\begin{tabular}{|c|c|c|}
\hline 90 & 130 & 145 \\
\hline 160 & 140 & 200 \\
\hline 100 & 140 & 250 \\
\hline
\end{tabular}

$3 \mathrm{X} 3$ neighborhood

$\mathrm{TU}=\{0,1,3,4,4,2,0,4\}$

Fig 4. Transformation model of a neighborhood to a Fuzzy Texture Unit(FTU).

Fuzzy Texture Spectrum(FTS) is termed as the frequency of distribution of all fuzzy texture units, with the abscissa indicating the texture unit number and the ordinate representing its occurrence frequency.

\subsection{Texture Spectrum}

The texture units describe the local-texture aspect of a given pixel; that is, the relative grey-level relationships between the central pixel and its neighbors. Thus the statistics of the frequency of occurrence of all the texture units over a large region of an image should reveal texture information. The texture spectrum is the frequency distribution of all the texture units, with the abscissa indicating the texture unit number $\mathrm{N}_{\mathrm{TU}}$ and the ordinate representing its occurrence frequency[3].

In practice, a real texture image is usually composed of two parts: Texture elements and random noise or background. The greater the proportion of texture components compared to the background, the better that texture can be perceived by human vision. In the texture spectrum the increase in percentage of texture feature components in an image will result in a tendency to form a particular distribution of peaks. In addition, different images are composed of particular texture units with different distributions in their texture spectra. In this way the texture of an image can be characterized by its texture spectrum.

It should be noted that the labeling method chosen may affect the relative positions of the texture units in the texture spectrum, but will not change their frequency values in the latter.

It should be also noted that the local texture for a given pixel and its neighborhood is characterized by the corresponding texture unit, while the texture aspect for a uniform texture image is revealed by its texture spectrum calculated within an appropriate window. The size of the window depends on the nature of the texture image. The texture spectrum has discriminating performance for different textures, because different texture images will have correspondingly different spectra, where as similar texture images will have correspondingly the similar spectra.

\section{EXPERIMENTAL RESULTS}

The proposed texture based similarity features are applied using IHBM Similarity Measures for efficient medical image retrieval system. The results are compared for the query image with database images. The principle of the similarity measure is the computation of the distance between the extracted texture features of the query image and those of the images in the database. Once all the distances are computed, the algorithm ranks the images of the database from the nearest to the furthest to the query image.

The above proposed methods are applied on 75 MRI and 25 Orthopedics medical images. The results of the query image and its retrieved relevant images of the proposed methods BTS, RTS and FTS using IHBM similarity measures are shown in the figures $5,6,7,8,9$ and 10 respectively. Table 1 and 2 shows the retrieved images and precision rate, which is nothing but the capability of the system to retrieve only the relevant images, for top 5 and 10 retrieved images for the query images 7 and 76 respectively.

From the Tables 1 and 2 it is clearly evident that the proposed FTS Texture Similarity Feature with IHBM Similarity Measures out performs with the other texture similarity features derived from TS, RTS. The precision $\mathrm{V}_{\mathrm{S}}$ the number of images returned for the above Tables is also plotted in the Figure 11 and 12.

Table 1: Retrieved relevant images for top 5 and 10 for image \#7

\begin{tabular}{|c|c|c|c|}
\hline $\begin{array}{c}\text { Query image } \\
\# 7\end{array}$ & BTS & RTS & FTS \\
\hline 5 & 4 & 4 & 4 \\
\hline 10 & 5 & 7 & 7 \\
\hline $\begin{array}{c}\text { Precision for Top 5and 10 images for query image } \\
\# \text { 7 } \\
\text { \# 7 }\end{array}$ & BTS & RTS & FTS \\
\hline 5 & 0.8 & 0.8 & 0.8 \\
\hline 10 & 0.5 & 0.7 & 0.7 \\
\hline
\end{tabular}

Table 2:Retrieved relevant images for top 5 and 10 for image

\begin{tabular}{|c|c|c|c|}
\hline & \multicolumn{3}{|c|}{$\# 76$} \\
\hline $\begin{array}{l}\text { Query image } \\
\# 76\end{array}$ & BTS & RTS & FTS \\
\hline 5 & 4 & 3 & 4 \\
\hline 10 & 7 & 4 & 9 \\
\hline \multicolumn{4}{|c|}{$\begin{array}{l}\text { Precision for Top } 5 \text { and } 10 \text { images for query image } \\
\text { \# } 76\end{array}$} \\
\hline $\begin{array}{l}\text { Query image } \\
\# 76\end{array}$ & BTS & RTS & FTS \\
\hline 5 & 0.8 & 0.6 & 0.8 \\
\hline 10 & 0.7 & 0.4 & 0.9 \\
\hline
\end{tabular}




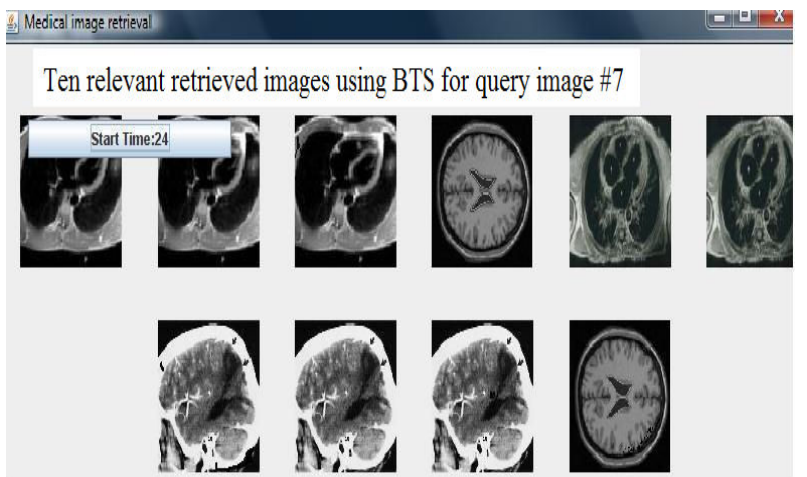

Fig 5: The results of the query image and ten retrieved relevant images using BTS for image \#7

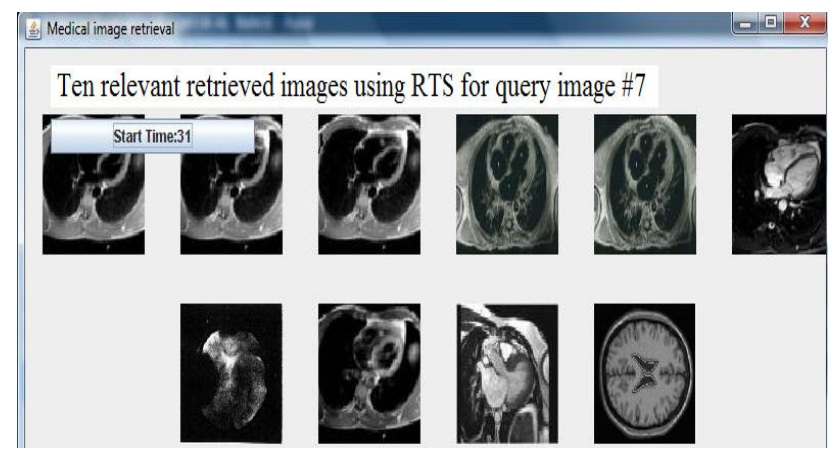

Fig 6: The results of the query image and ten retrieved relevant images using RTS for image \#7

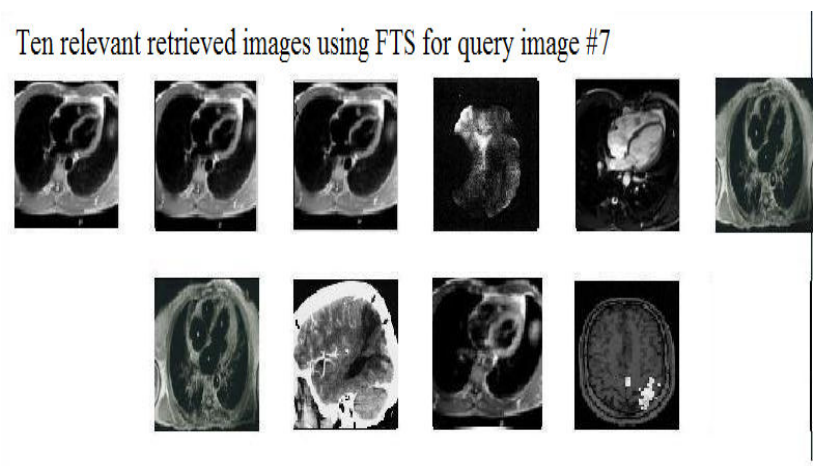

Fig 7: The results of the query image and ten retrieved relevant images using FTS for image \#7

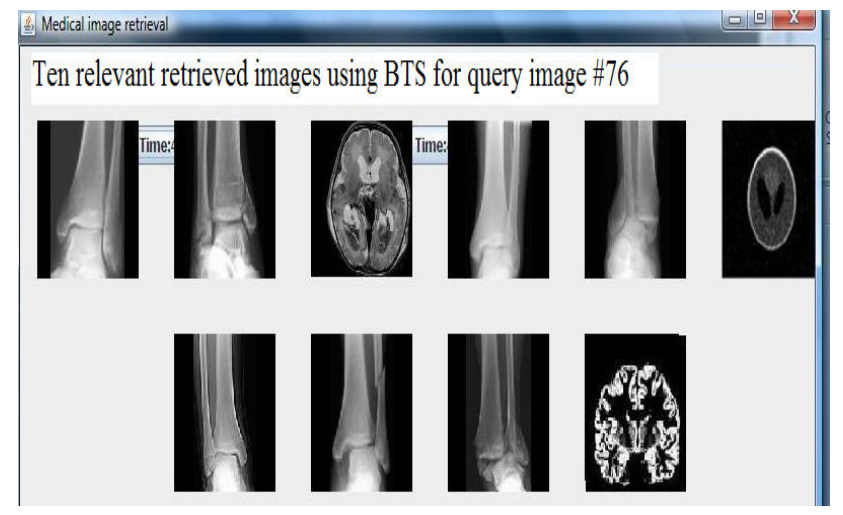

Fig 8: The results of the query image and ten retrieved relevant images using BTS for image \#76

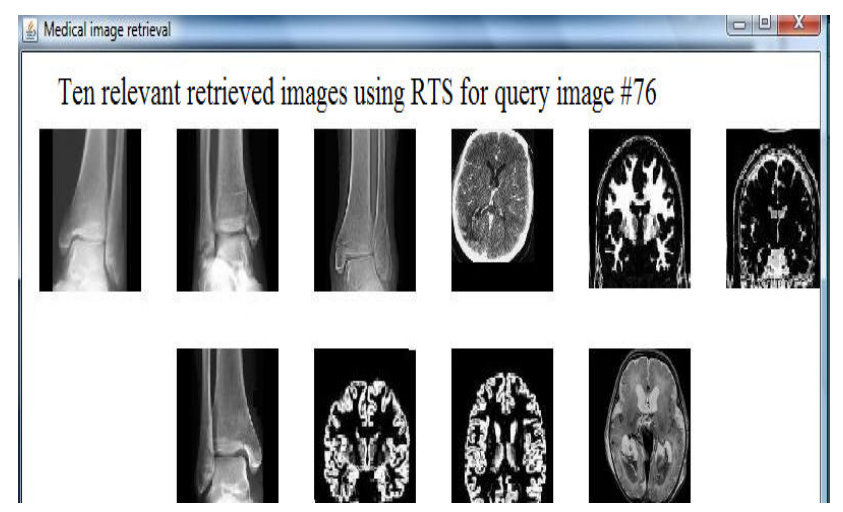

Fig 9: The results of the query image and ten retrieved relevant images using RTS for image \#76

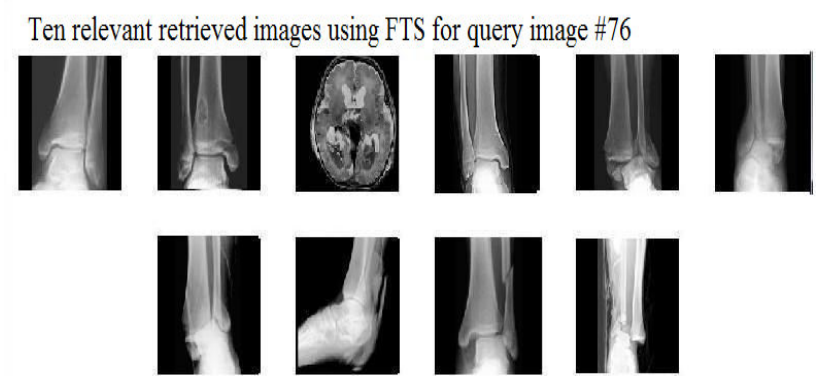

Fig 10:The results of the query image and ten retrieved relevant images using FTS for image \#76 


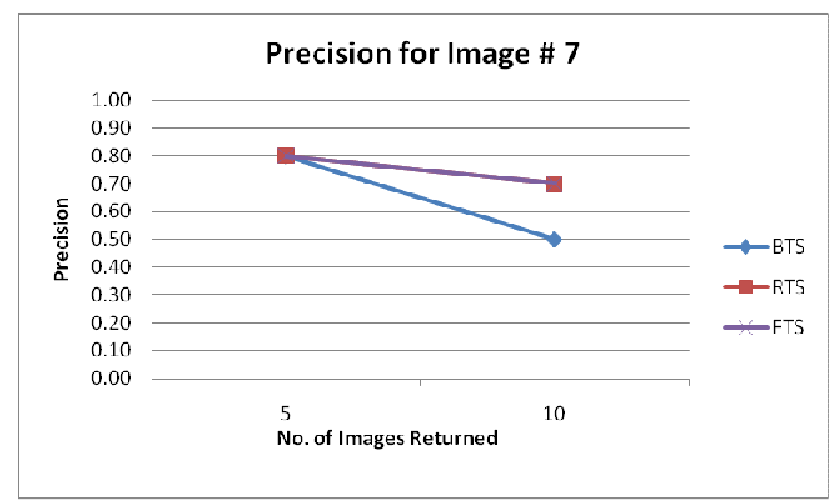

Fig 11: Precision $V_{s}$ No. of Images returned for image \#7

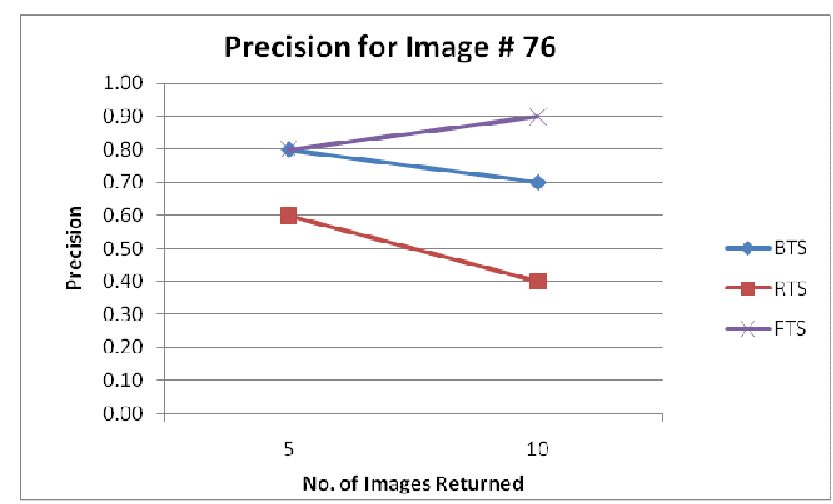

Fig 12: Precision $V_{s}$ No. of Images returned for image \#76

\section{CONCLUSIONS}

For building effective image retrieval system, especially with different medical images of noise, poor resolution and illumination levels, present paper derived three efficient texture based similarity features called BTS, RTS and FTS. The experimental results prove that, all the three proposed Texture similarity features with IHBM Similarity Measures have shown good performance in retrieving the similar medical images. The three Texture similarity features are compared. The BTS is more complex in nature due to its high range of TU values and also shown poor performance in image retrieval. The FTS has shown good retrieved instances and precision rate than other two measures. The complexity of FTS ranges in between BTS and RTS. The RTS in some cases has shown the same retrieval performance as FTS and its complexity is least when compared to other two measures.

\section{ACKNOWLEDGEMENTS}

The authors wish to express their sincere thanks to K.V.V. Satya Narayana Raju, Chairman, Chaitanya Institutions and K. Sashi Kiran Varma, Secretary, GIET, Rajahmundry for providing Research facilities at SRRF Labs. and the authors sincerely acknowledge moral support extended by Dr. Nageswara Rao Vallurupalli, Chairman, and Sri. Satyanarayana Rao Vallurupalli, Secretary\& Correspondent, Gudlavalleru Engineering College, Gudlavalleru. Authors would like to thank Dr. G.V.S. Anantha Lakshmi for her invaluable suggestions and constant encouragement that led to improvise the presentation quality of the paper.

\section{REFERENCES}

[1] R. Haralick, K. Shanmugam, and I. Dinstein. Textural features for image classifcation. IEEE Transactions Systems on Man and Cybernetics, 3(6):610 621,1973.

[2] Amith k Bhattacharya, P K Srivatsava and Anil Bhagat., a modified Texture filter for Satellite images, $22^{\text {nd }}$ Asian Conference on Remote Sensing,5-9 November 2001,Singapore.

[3] He, D.C., and L.Wang, 1990. Texture unit, texture spectrum and texture analysis. IEEE Trans. Geoscience Remote Sensing, 28(4), pp.509-512

[4] Wang, L. and He, D.C. Texture Classification Using Texture Spectrum, Pattern Recognition, Vol. 23, pp. 905-910, 1990

[5] Van Gool, L., Dewaele, P. and Oosterlinck, A. Surveytexture analysis anno 1983, Computer Vision, Graphics Image Processing, Vol. 29, PP. 336-357, 1985.

[6] Salari, E. and Ling, Z. Texture segmentation using hierarchical wavelet decomposition, Patt. Recogn. 28, 12, pp.1819-1824, 1995.

[7] Bovik, A. C., Clark, M. and Geisler, W. S. Multichannel texture analysis using localized spatial filters, IEEE Trans. Patt. Anal. Mach. Intell., 12, 1, pp. 55-73, 1990.

[8] Chang. T, and Kuo, C. C. J., Texture analysis and classification with tree-structured wavelet transform, IEEE Trans. Image Processing, 2, 4, pp. 429-442, 1993.

[9] Chen, J. L. and Kundu, Unsupervised texture segmentation using multi-channel decomposition and hidden Markov models, IEEE Trans. Image. Processing, 4, 5, pp. 603-620, 1995.

[10] Haralick, R. M. Statistical and structural approaches to texture, Proc. of 4th Int. Joint Conf. Pattern Recognition, pp. $45-60,1979$.

[11] He, D. C. and Wang, L. Textural filters based on the texture spectrum, Patt. Recogn. 24, 12, pp.1187-1195, 1991.

[12] He, D.C. and Li, Wang. Texture Features Based on Texture Spectrum, Pattern Recognition, Vol. 24, pp. 391-399, 1991.

[13] H.Müller, N.Michous, D.Bandon and A.Geissbuhler. A review of content-based image retrieval systems in medical applications - clinical benefits and future directions. International Journal of Medical Informatics, 73(1):23,Feb, 2004.

[14] A. Kak and C. Pavlopoulou. Content-Based Image Retrieval from Large Medical Databases. In 3D Data Processing, Visualization, Transmission, Padova, Italy, June 2002.

[15] F. Korn, N. Sidiropoulos, C. Faloustos, E. Siegel, and Z. Protopapas. Fast and effective retrieval of medical tumor shapes. IEEE Transactions on Knowledge and Data Engineering, 10(6):889-904, 1998. 
[16] T. Lehmann, B. Wein, J. Dahmen, J. Bredno,F. Vogelsang, and M. Kohnen. Content-Based Image Retrieval in Medical Applications : A Novel Multi-Step Approach. In International Society for Optical Engineering (SPIE), volume 3972(32), pages 312-320, Feb. 2000.

[17] H. D. Tagare, C. C. Jaffe, and J. Duncan. Medical Image Databases: A Content-based Retrieval Approach. J Am Med Inform Assoc, 4(3):184-198,1997.

[18] Tristan Glatard, Johan Montagnat, Isabelle E. Magnin CREATIS (CNRS-Inserm), INSA, Texture Based Medical Image Indexing and Retrieval: Application to Cardiac Imaging, MIR'04, October 15-16, 2004, New York, New York, USA

[19] Vijaya Kumar N. Gnaneswara Rao, A.L.Narsimha Rao, V.Venkata Krishna, IHBM: Integrated Histogram Bin Matching For Similarity Measures of Color Image Retrieval, International Journal of Signal Processing, Image Processing and Pattern Recognition,IJSIP, Vol. 2, No. 3, September, 2009

[20] M. Flickner, H. Sawhney, W. Niblack, J. Ashley, Q. Huang, B. Dom, M. Gorkani, J. Hafner, D. Lee,D. Petkovic, D. Steele, and P. Yanker. Query by image and video content : The QBIC system. IEEE Computer, 28(9):23-32, 1995.

[21] Vijaya Kumar N. Gnaneswara Rao, A.L.Narsimha Rao, RTL: Reduced Texture spectrum with Lag value Based Image Retrieval for Medical Images, International Journal of Future Generation Communication and Networking Vol. 2, No. 4, December, 2009

\section{AUTHORS PROFILE}

N.Gnaneswara Rao received B.E. (ECE) degree from Andhra University (S.R.K.R. Engineering College) in 1996 and received his M.Tech (CS) post graduation from the JNTU Hyderabad in 2002. He is pursuing PhD at JNTU Kakinada in the area of Content Based Image Retrieval. He has been working in Gudlavalleru Engg College since 2000. He is a life member for CSI, ISTE, CRSI, IETE and a member of ACM, IET, and IEEE. He has published Ten research publications in various National, and International conferences
Dr. Vakulabharanam Vijaya Kumar received integrated M.S. Engg, degree from Tashkent Polytechnic Institute (USSR) in 1989. He received his Ph.D. degree in Computer Science from Jawaharlal Nehru Technological University (JNTU) in 1998.He has served the JNT University for 13 years as Assistant Professor and Associate Professor and taught courses for M.Tech students. He has been Dean for Dept of CSE and IT at Godavari Institute of Engineering and Technology since April, 2007.His research interests includes Image Processing, Pattern Recognition, Digital Water Marking and Image Retrieval Systems. He is a life member for CSI, ISTE, IE, IRS, ACS and CS. He has published more than 120 research publications in various National, International conferences, proceedings and Journals. He has established Srinivasa Ramanujan Research Forum (SRRF) at GIET, Rajahmundry, India for promoting research activities.

Dr. P.S.V.Srinivasa Rao received his Ph.D. degree in Computer Science.He is having more than 17 years of experience He has been serving as prinicpal at Rao \& Naidu Engineering College Ongole. His research interest is on the area of Image Processing. He is a life member for ISTE. He has published a book and he is having more than 5 research publications in various National, International conferences, proceedings and Journals. 